Résumés des conférences et travaux

\title{
Histoire de la Mésopotamie
}

\section{Dominique Charpin}

\section{OpenEdition \\ Journals}

Édition électronique

URL : https://journals.openedition.org/ashp/1254

DOI : $10.4000 /$ ashp. 1254

ISSN : 1969-6310

Éditeur

Publications de l'École Pratique des Hautes Études

Édition imprimée

Date de publication : 1 octobre 2012

Pagination : 10-11

ISSN : 0766-0677

Référence électronique

Dominique Charpin, « Histoire de la Mésopotamie », Annuaire de l'École pratique des hautes études (EPHE), Section des sciences historiques et philologiques [En ligne], 143 | 2012, mis en ligne le 20 septembre 2012, consulté le 03 août 2021. URL : http://journals.openedition.org/ashp/1254 ; DOI : https://doi.org/10.4000/ashp.1254 


\title{
HISTOIRE DE LA MÉSOPOTAMIE
}

\author{
Directeur d'études : M. Dominique CHARPIN
}

Programme de l'année 2010-2011 : Recherches sur les archives paléo-babyloniennes (suite).

Comme les années précédentes, l'intitulé du programme annoncé était volontairement assez général : il s'agissait d'étudier des documents d'archives d'époque paléobabylonienne en liaison avec le projet ANR «ARCHIBAB » (Archives babyloniennes, $\mathrm{XX}^{\mathrm{e}}-\mathrm{XVII}^{\mathrm{e}}$ siècles av. J.-C.). La parution dans l'été 2010 de l'ouvrage posthume de A. Goetze, Cuneiform Texts from Various Collections (YOS 15, New Haven - Londres), a été l'occasion de compléter plusieurs dossiers déjà connus. Dans ce recueil ont été publiées les copies de 217 tablettes conservées dans diverses collections américaines, publiques et privées, que l'illustre assyriologue américain avait accumulées au cours de ses déplacements dans de nombreuses villes. Les difficultés qu'a eues l'éditeur du volume, B. Foster, pour retrouver la trace de certaines de ces collections - et ses échecs dans plus d'un cas - illustrent l'un des problèmes que posent les collections privées ou semi-privées (on songe à certains Colleges qui n'ont pas hésité à vendre les tablettes de leurs collections, sans garder trace de leur devenir).

Ce recueil comprend des textes couvrant les trois millénaires de l'histoire mésopotamienne : on s'est limité à l'examen des 81 textes d'archives d'époque paléo-babylonienne. Selon la tradition de la série YOS, les copies des originaux sont accompagnées d'une introduction (p. 3-10) d'un catalogue (p. 12-19), et d'index, le tout préparé en l'occurrence par les soins de K. R. Veenhof; cependant, aucune édition des documents n'est donnée. Les éditions préparées à l'occasion de la conférence de cette année seront bientôt mises à la disposition de tous sur le site www.archibab.fr.

Ce sont les lettres qui constituent le plus gros lot: pas moins de 55 nouveaux textes. Quatre émanent du roi de Larsa Rim-Sin ( $\left.{ }^{\text {os }} 20-23\right)$. Le lot le plus important est celui de la correspondance passive de Šamaš-hazir. Ce personnage fut placé par le roi Hammu-rabi de Babylone à la tête du domaine royal dans l'ancien royaume de Larsa après son annexion, avec le titre de šassukkum. Quatorze lettres supplémentaires ( ${ }^{\text {os }}$ 24-37) viennent compléter un dossier déjà fourni. Elles permettent de mieux connaître les procédures, notamment dans l'attribution des « champs alimentaires », ou dans l'administration du réseau d'irrigation, crucial pour l'agriculture dans une région sèche comme le sud de l'Irak actuel. On trouve aussi des informations relatives aux instruments servant à cette irrigation (avec peut-être un nouveau terme, nașbûm, au $\mathrm{n}^{0} 39: 5$; cf. 1. 8). On notera également au $\mathrm{n}^{\circ} 27$ l'allusion à un édit royal (șimdatum) jusque là inconnu, portant sur le partage de la récolte entre cultivateur et possesseur d'un champ; la part de chacun lors de la moisson y était manifestement fixée. Grâce au n ${ }^{\circ} 28$, une réinterprétation de $A b B 4166$ (copie désormais publiée dans OECT 15 75) est possible; cette lettre, dont l'adresse a disparu, fut manifestement adressée par Hammu-rabi à Šamaš-hazir et Marduk-nașir. 
Les lettres nos $38-45$ proviennent de la ville de Lagaba. Cette localité n'a malheureusement fait l'objet que de fouilles irrégulières, de sorte que sa localisation précise reste inconnue. Les archives découvertes datent du règne de Samsu-iluna. Ces lettres ont été étudiées par A. Jacquet, qui prépare actuellement l'édition de nombreux autres textes de cette localité conservés à l'université de Yale. On relève en particulier des lettres d'une femme nommée Unnubtum, qui fut manifestement une religieusenadîtum de Marduk à Babylone (cf. no 48; voir L. Barberon, Archibab 1. Les religieuses et le culte de Marduk dans le royaume de Babylone (19 $-17^{\mathrm{e}}$ siècles av. n.è.), Mémoires de NABU 14, Paris, sous presse).

Trois lettres viennent compléter le dossier d'Alammuš-nașir, étudié en 2006-2007 (cf. Annuaire de l'EPHE SHP 139, 2006-2007, p. 17-19). Parmi celles-ci, le no 65 détaille les mesures de rétorsion prises contre la famille d'un débiteur insolvable qui a pris la fuite. La note finale, qui mentionne des juments ( ${ }^{m u n u s}$ ANŠE.KUR.RA.HI.A 1. 26) est également très intéressante, en raison de la rareté des mentions de chevaux à cette époque (cf. le document, contemporain, de Tell Leilan, publiée par A. Tsukimoto, «From Lullû to Ebla. An Old Babylonian Document Concerning a Shipment of Horses », dans B. Pongratz-Leisten et al. (éd.), Ana šadî Labnāni lū allik. Beiträge zu altorientalischen und mittelmeerischen Kulturen. Festschrift für Wolfgang Röllig, Neukirchen - Vluyn, 1997 [AOAT 247], p. 407-412). Il y a également des mentions de chevaux dans les documents de Me-Turan qui datent du règne d'Iluni, contemporain de Samsu-iluna (cf. A. Mustafa, The Old Babylonian Tablets from Me-Turan [Tell al-Sib and Tell Haddad], Diss. University of Glasgow, 1983). Il est donc très vraisemblable qu'à l'issue de la campagne de Samsu-iluna contre Ešnunna, Alammuš-nașir reçut non seulement des esclaves ešnunnéennes ( $A b B 9$ 127), mais aussi des chevaux.

Enfin, les textes $\mathrm{n}^{\text {os }} 72-96$ sont des contrats et des textes administratifs, qui complètent également des dossiers déjà connus, dont certaines pièces ont également été lues lors des conférences. Le $\mathrm{n}^{\circ} 85$ est un prêt d'argent pour lequel le débiteur laisse une maison en gage (šipirtum 1. 4 ; cf. ma note dans NABU 2009/59); la clause relative au remboursement ne mentionne pas « When paid in time (?) » (catalogue de YOS 15, p. 17), mais « au mois de sibûtum (en lisant ITI si-bu-ti-im; cf. ma note dans $N A B U$ 1989/93). L'étude du no 96 a permis à B. Fiette, doctorant à la Section, de publier une très intéressante note (« Du nouveau à propos de la mainmise babylonienne sur les biens du palais de Larsa (YOS 15 96)», NABU 2011/17). 